\title{
关于 Teichmüller 极值的等价性
}

范金华*，陈纪修

复旦大学数学科学学院, 上海 200433

*E-mail: jinhuafan@hotmail.com

收稿日期: 2007-10-12；接受日期: 2008-03-14

国家自然科学基金 (批准号: 10571028) 资助项目

摘要 分别记 $T(\triangle)$ 与 $B(\triangle)$ 为单位圆盘 $\triangle$ 上的 Teichmüller 空间与无限小 Teichmüller 空间. 证明了 $[\nu]_{B(\triangle)}$ 是无限小 Strebel 点并不能说明 $[\nu]_{T(\triangle)}$ 是一个 Strebel 点以及 $[\nu]_{T(\triangle)}$ 是 Strebel 点并不能说明 $[\nu]_{B(\triangle)}$ 是一个无限小 Strebel 点. 作为这个结论的应用, 解决了姚 国武提出的问题.

关键词 Teichmüller 空间 无限小 Teichmüller 空间 Strebel 点 $\operatorname{MSC}(2000)$ 主题分类 $30 \mathrm{C} 75$

\section{1 引言}

令 $\mathcal{D}$ 是复平面 $\mathbb{C}$ 上一个边界至少两点的区域, $M(\mathcal{D})$ 为 $L^{\infty}(\mathcal{D})$ 中开的单位球. $M(\mathcal{D})$ 中的元素 $\mu$ 诱导 $\mathcal{D}$ 上的拟共形映射 $f$, 使得 $f$ 满足 Beltrami 方程

$$
f_{\bar{z}}(z)=\mu(z) f_{z}(z) .
$$

反之, $\mathcal{D}$ 上的拟共形映射 $f$ 有一个 Beltrami 系数 (或称为复特征) $\mu_{f}(z)=f_{\bar{z}}(z) / f_{z}(z) \in$ $M(\mathcal{D})$ 与之对应. $M(\mathcal{D})$ 中的两个 Beltrami 系数 $\mu$ 和 $\nu$ 称为等价的, 记为 $\mu \sim \nu$, 如果它们 满足下列条件: 假设由 $\mu$ 和 $\nu$ 所诱导的拟共形映射分别为 $f$ 和 $g$, 如果存在 $f(\mathcal{D})$ 到 $g(\mathcal{D})$ 的共形映射 $c$ 以及 $\mathcal{D}$ 到 $\mathcal{D}$ 的一个拟共形合痕 $h_{t}(0 \leqslant t \leqslant 1)$, 使得

1. $h_{0}$ 是 $\mathcal{D}$ 上的恒等映射,

2. $h_{1}=g^{-1} \circ c \circ f$,

3. 对每个 $t \in[0,1]$ 以及 $\mathcal{D}$ 的任意理想边界点 $p$ 都成立 $h_{t}(p)=g^{-1} \circ c \circ f(p)$.

上述等价关系将集合 $M(\mathcal{D})$ 分为一些等价类, 这些等价类的全体所组成的集合称为 $\mathcal{D}$ 上的 Teichmüller 空间, 记为 $T(\mathcal{D})$.

对于 $M(\mathcal{D})$ 中的一个 Beltrami 系数 $\mu$, 我们记 $[\mu]_{T(\mathcal{D})}$ 为 $M(\mathcal{D})$ 中所有与 $\mu$ 等价的 Beltrami 系数全体, 令

$$
k_{0}\left([\mu]_{T(\mathcal{D})}\right)=\inf \left\{\|\nu\|_{\infty}, \nu \in[\mu]_{T(\mathcal{D})}\right\} .
$$


如果 $\|\mu\|_{\infty}=k_{0}\left([\mu]_{T(\mathcal{D})}\right)$, 则称 $\mu$ 在 $T(\mathcal{D})$ 中极值; 如果对 $[\mu]_{T(\mathcal{D})}$ 中的任意其他元素 $\nu$ 都成 立 $\|\nu\|_{\infty}>\|\mu\|_{\infty}$, 则称 $\mu$ 在 $T(\mathcal{D})$ 中唯一极值. 利用拟共形映射的紧性, 我们知道在 $[\mu]_{T(\mathcal{D})}$ 中至少存在一个极值 Beltrami 系数.

令 $A(\mathcal{D})$ 为 $\mathcal{D}$ 上的全体全纯函数 $\phi(z)$ 且

$$
\|\phi\|=\iint_{\mathcal{D}}|\phi(z)| d x d y<\infty,
$$

记 $A_{1}(\mathcal{D})$ 为 $A(\mathcal{D})$ 中的单位球面. Hamilton ${ }^{[1]}$, Krushkal ${ }^{[2]}$, Riech 和 Strebel ${ }^{[3]}$ 证明了一个 Beltrami 系数 $\mu$ 在 $T(\mathcal{D})$ 中极值的充要条件是

$$
\|\mu\|_{\infty}=\sup _{\varphi \in A_{1}(\mathcal{D})}\left|\iint_{\mathcal{D}} \varphi \mu d x d y\right| .
$$

假设 $\mu$ 和 $\nu$ 为 $L^{\infty}(\mathcal{D})$ 中的两个元素, 如果对 $A(\mathcal{D})$ 中的任意元素 $\phi$ 都成立 $\iint_{\mathcal{D}} \mu \phi=$ $\iint_{\mathcal{D}} \nu \phi$, 则称 $\mu$ 和 $\nu$ 为无限小等价的, 记为 $\mu \approx \nu$. 这种等价关系将 $L^{\infty}(\mathcal{D})$ 中的元素分 为一些等价类, 这些等价类的全体所组成的集合称为 $\mathcal{D}$ 上的无限小 Teichmüller 空间, 记为 $B(\mathcal{D})$. 我们知道 $B(\mathcal{D})$ 是 $T(\mathcal{D})$ 在基点处的切空间.

对 $L^{\infty}(\mathcal{D})$ 中的元素 $\mu$, 记 $[\mu]_{B(\mathcal{D})}$ 为 $L^{\infty}(\mathcal{D})$ 中所有与 $\mu$ 无限小等价的元素全体, 令

$$
\|\mu\|=\inf \left\{\|\nu\|_{\infty}, \nu \in[\mu]_{B(\mathcal{D})}\right\} .
$$

如果 $\|\mu\|_{\infty}=\|\mu\|$, 则称 $\mu$ 在 $B(\mathcal{D})$ 中极值; 如果对 $[\mu]_{B(\mathcal{D})}$ 中的任意其他元素 $\nu$ 都成立 $\|\nu\|_{\infty}>\|\mu\|_{\infty}$, 则称 $\mu$ 在 $B(\mathcal{D})$ 中唯一极值.

$\mu$ 在 $T(\mathcal{D})$ 中极值等价于 $\mu$ 在 $B(\mathcal{D})$ 中极值. 由于 Božin ${ }^{[4]}$ 等人的重要工作, 我们知道 上述等价性对于唯一极值也是成立的.

对于 $M(\mathcal{D})$ 中元素 $\mu$, 定义 $h\left([\mu]_{T(\mathcal{D})}\right)$ 为

$$
h\left([\mu]_{T(\mathcal{D})}\right)=\inf \left\{\left\|\left.\nu\right|_{\mathcal{D} \backslash F}\right\|_{\infty}: \nu \sim \mu, F \text { 是 } \mathcal{D} \text { 的紧子集 }\right\},
$$

则显然有 $h\left([\mu]_{T(\mathcal{D})}\right) \leqslant k_{0}\left([\mu]_{T(\mathcal{D})}\right)$. 按照文献 $[5,6]$ 的定义, 如果 $h\left([\mu]_{T(\mathcal{D})}\right)<k_{0}\left([\mu]_{T(\mathcal{D})}\right)$, 则称 $[\mu]_{T(\mathcal{D})}$ 为 Strebel 点, 否则称 $[\mu]_{T(\mathcal{D})}$ 为非 Strebel 点. 根据 Strebel 标架准则 ${ }^{[6]}$, 任意 Strebel 点 $[\mu]_{T(\mathcal{D})}$ 中必含有唯一极值 Beltrami 系数 $\nu=k \frac{\bar{\varphi}}{|\varphi|}$, 其中 $k=k_{0}\left([\mu]_{T(\mathcal{D})}\right), \varphi \in A_{1}(\mathcal{D})$.

类似于 $h\left([\mu]_{T(\mathcal{D})}\right)$ 的定义, 我们可以定义边界半模 $b([\mu])$ 为

$$
b([\mu])=\inf \left\{\left\|\left.\nu\right|_{\mathcal{D} \backslash F}\right\|_{\infty}: \nu \approx \mu, F \text { 是 } \mathcal{D} \text { 的紧子集 }\right\} .
$$

如果 $\|\mu\|>b([\mu])$, 则称 $[\mu]_{B(\mathcal{D})}$ 为无限小 Strebel 点, 否则称 $[\mu]_{B(\mathcal{D})}$ 为无限小非 Strebel 点. 根据无限小 Strebel 标架准则, 任意无限小 Strebel 点 $[\mu]_{B(\mathcal{D})}$ 中必含有唯一极值 Beltrami 系 数 $\nu=k \frac{\bar{\varphi}}{\varphi \mid}$, 其中 $k=\|\mu\|, \varphi \in A_{1}(\mathcal{D})$.

在拟共形极值理论的研究中, Strebel 点发挥了重要的作用. 例如利用 Strebel 点, Božin 等人 [4] 给出了唯一极值 Beltrami 系数特征的刻画; 在文献 [7] 中, Strebel 利用点移动微分给 出了一种构造 Hamilton 序列的方法. Strebel 点在 Teichmüller 空间中也具有许多特殊的几何 和解析性质, 例如当 $\tau$ 是一个 Strebel 点, 则存在唯一一条测地线连接基点与 $\tau^{[8]}$, Teichmüller 距离函数在 $\tau$ 点是连续可微的 ${ }^{[5]}$. 其他关于 Strebel 点与极值拟共形映射的性质, 可以参考 文献 $[5-7,9,10]$.

$\mu$ 在 $T(\mathcal{D})$ 中极值 (唯一极值) 等价于 $\mu$ 在 $B(\mathcal{D})$ 中极值 (唯一极值), 由于 Strebel 点在 拟共形映射极值理论研究中的重要地位, 我们问以上等价关系对 Strebel 点是否成立. 如果 
假设 $\mu$ 是极值的, 则知道 $[\mu]_{T(\mathcal{D})}$ 是 Strebel 点等价于 $[\mu]_{B(\mathcal{D})}$ 是无限小 Strebel 点. 但是对 于一般的情形, 我们还不知道这个等价关系是否成立. 本文, 给出这个问题否定的回答.

记 $\triangle$ 是复平面 $C$ 上的单位圆盘, 在本文中我们限定考虑情形 $\mathcal{D}=\triangle$.

定理 1 存在 $\nu \in M(\triangle)$, 使得 $[\nu]_{T(\triangle)}$ 是一个非 Strebel 点, 但是 $[\nu]_{B(\triangle)}$ 是一个无限 小 Strebel 点.

定理 2 存在 $\nu \in M(\triangle)$, 使得 $[\nu]_{B(\triangle)}$ 是一个无限小非 Strebel 点, 但是 $[\nu]_{T(\triangle)}$ 是一 个 Strebel 点.

利用定理 1 和 2 , 我们将解决姚国武在文献 $[11,12]$ 中提出的一些问题.

\section{2 非常数模极值 Beltrami 系数}

称一个 Beltrami 系数 $\mu(z) \in M(\triangle)$ 是 Teichmüller Beltrami 系数, 如果它具有形式

$$
\mu(z)=k \frac{\varphi(z)}{|\varphi(z)|}, \text { a.e. } z \in \triangle,
$$

其中 $\varphi(z)$ 是 $\triangle$ 上的全纯函数, $0 \leqslant k<1$ 是一个常数. 在 Božin 等人的文章 [4] 和 Reich 的 文章 ${ }^{[13]}$ 之前, 所知的唯一极值 Beltrami 系数 $\mu(z)$ 都是 Teichmüller Beltrami 系数, 从而具 有常数模, 即

$$
|\mu(z)|=\text { const, a.e. } z \in \triangle \text {. }
$$

因此很长一段时间人们都猜想唯一极值 Beltrami 系数 $\mu(z)$ 必然具有常数模, 或者 $\mu(z)$ 必 然具有形式 $(2.1)$. 文献 $[4,13]$ 否定了这个猜想.

定理 $\mathbf{A}^{[4,13]}$ 在 $T(\triangle)$ 中存在唯一极值 Beltrami 系数 $\mu(z)$, 使得 $|\mu(z)|$ 在上 $\triangle$ 不是常 数.

定理 $\mathbf{B}^{[4]}$ 在 $T(\triangle)$ 中存在唯一极值 Beltrami 系数 $\mu(z)$, 在 $\triangle$ 上几乎处处成立 $|\mu(z)| \neq$ $\|\mu\|_{\infty}$.

代替文献 $[4,13]$ 中对唯一极值 Beltrami 系数的考虑, 姚国武证明了

定理 $\mathbf{C}^{[11]}$ 在 $M(\triangle)$ 中存在 Beltrami 系数 $\mu(z)$, 使得 $[\mu]_{T(\triangle)}\left([\mu]_{B(\triangle)}\right)$ 包含无穷多个 极值 Beltrami 系数, 但 $[\mu]_{T(\Delta)}\left([\mu]_{B(\Delta)}\right)$ 中不包含极值 Teichmüller Beltrami 系数.

定理 $\mathbf{D}^{[12]}$ 在 $M(\triangle)$ 中存在 Beltrami 系数 $\mu(z)$, 使得 $[\mu]_{T(\triangle)}\left([\mu]_{B(\triangle)}\right)$ 包含无穷多个 极值 Beltrami 系数但 $[\mu]_{T(\triangle)}\left([\mu]_{B(\triangle)}\right)$ 中不包含常数模极值 Beltrami 系数.

姚国武 ${ }^{[11,12]}$ 提出了以下一些公开问题:

问题 $1^{[11]}[\mu]_{T(\triangle)}$ 中存在极值 Teichmüller Beltrami 系数是否意味着 $[\mu]_{B(\triangle)}$ 中存在 极值 Teichmüller Beltrami 系数, 反之如何?

问题 2 ${ }^{[12]}[\mu]_{T(\triangle)}$ 中存在常数模极值 Beltrami 系数是否意味着 $[\mu]_{B(\triangle)}$ 中存在常数模 极值 Beltrami 系数, 反之如何?

利用定理 1 和 2 , 我们通过下面两个定理否定地回答了问题 1 和 2 .

定理 3 存在 $\nu \in M(\triangle)$, 使得 $[\nu]_{T(\triangle)}$ 中不包含常数模极值 Beltrami 系数, 但是 $[\nu]_{B(\triangle)}$ 中包含唯一极值 Teichmüller Beltrami 系数.

定理 4 存在 $\nu \in M(\triangle)$, 使得 $[\nu]_{T(\triangle)}$ 中不包含常数模极值 Beltrami 系数, 但是 $[\nu]_{T(\triangle)}$ 中包含唯一极值 Teichmüller Beltrami 系数. 
从文献 [8] 我们知道在 $T(\triangle)$ 只有一条测地线连接基点和 $[\mu]_{T(\triangle)}$ 的充要条件是 $[\mu]_{T(\triangle)}$ 中包含唯一极值 Beltrami 系数, 且这个极值 Beltrami 系数具有常数模. 文献 [14] 证明在 $B(\triangle)$ 中也有相似的结论. 由定理 3 和 4 , 令 $\nu \in M(\triangle)$, 则下面推论是显然的.

推论 1 在 $T(\triangle)$ 中只有唯一的测地线连接 $[0]_{T(\triangle)}$ 和 $[\nu]_{T(\triangle)}$, 不能说明在 $B(\triangle)$ 中只 有唯一的测地线连接 $[0]_{B(\triangle)}$ 和 $[\nu]_{B(\triangle)}$.

推论 2 在 $B(\triangle)$ 中只有唯一的测地线连接 $[0]_{B(\triangle)}$ 和 $[\nu]_{B(\triangle)}$, 不能说明在 $T(\triangle)$ 中只 有唯一的测地线连接 $[0]_{T(\triangle)}$ 和 $[\nu]_{T(\triangle)}$.

\section{3 定理 1 的证明}

记 $\triangle_{r}=\{z \in \mathcal{C}:|z|<r\}$.

引理 1 令 $h(z)=z-\rho\left(\frac{1}{2}-|z|\right)$, 其中 $-1<\rho<1$, 则 $h(z)$ 是 $\triangle_{\frac{1}{2}}$ 到自身的拟共形映 射且满足 $\left.h(z)\right|_{\partial \triangle_{\frac{1}{2}}}=\mathrm{id}$.

证明 容易验证 $\left.h(z)\right|_{\partial \triangle_{\frac{1}{2}}}=\mathrm{id}$ 以及

对任意 $\rho \in(-1,1)$, 得到

$$
\frac{h_{\bar{z}}}{h_{z}}=\frac{\rho z^{\frac{1}{2}} \bar{z}^{-\frac{1}{2}}}{2+\rho z^{-\frac{1}{2}} \bar{z}^{\frac{1}{2}}}=\frac{\rho z}{2|z|+\rho \bar{z}}
$$

$$
\left\|\frac{h_{\bar{z}}}{h_{z}}\right\|_{\infty}<1, \quad h(0)=-\frac{1}{2} \rho \in \triangle_{\frac{1}{2}}
$$

因此 $h(z)$ 是 $\triangle_{\frac{1}{2}}$ 到自身的拟共形映射且满足 $\left.h(z)\right|_{\partial \triangle_{\frac{1}{2}}}=\mathrm{id}$, 证明完毕.

令 $\mu \in M(\triangle)$ 为一个极值 Beltrami 系数且满足: $\|\mu\|_{\infty}=k$, 当 $z \in \triangle_{\frac{1}{2}}$ 时 $\mu(z)=0$. 记 $f(z)$ 是 $\triangle$ 到 $\triangle$ 且以 $\mu$ 为复特征的拟共形映射. 作

记 $g(z)$ 的复特征为 $\nu(z)$, 则

$$
g(z)= \begin{cases}f(z), & z \in \triangle \backslash \triangle_{\frac{1}{2}}, \\ f \circ h(z), & z \in \triangle_{\frac{1}{2}} .\end{cases}
$$

$$
\nu(z)= \begin{cases}\mu(z), & z \in \triangle \backslash_{\frac{1}{2}}, \\ \frac{\rho z}{2|z|+\rho \bar{z}}, & z \in \triangle_{\frac{1}{2}} .\end{cases}
$$

从映射 $f(z)$ 和 $g(z)$ 的定义中不难看出 $[\mu]_{T(\Delta)}=[\nu]_{T(\Delta)}$ 且 $[\nu]_{T(\triangle)}$ 是一个非 Strebel 点.

定理 1 的证明 令 $\nu(z)$ 为 (3.2) 式所构造. 对一个给定的 $\rho \in(-1,1)$ 且 $\rho \neq 0$, 将要 证明当 $\|\mu\|_{\infty}=k<\frac{1}{28} \rho^{2}$ 时, $[\nu]_{B(\triangle)}$ 是一个无限小 Strebel 点. 从 $b([\nu])$ 的定义中不难看出 $b([\nu]) \leqslant k$. 令 $\eta(z)$ 是 $[\nu]_{B(\triangle)}$ 中的一个极值 Beltrami 系数, 则

$$
\begin{aligned}
\|\nu\| & =\|\eta\|_{\infty}=\sup _{\varphi \in A_{1}(\triangle)}\left|\iint_{\triangle} \eta(z) \varphi(z) d x d y\right| \\
& =\sup _{\varphi \in A_{1}(\triangle)}\left|\iint_{\triangle} \nu(z) \varphi(z) d x d y\right| \geqslant \frac{1}{\pi}\left|\iint_{\triangle} \nu(z) d x d y\right| \\
& =\frac{1}{\pi}\left|\iint_{\triangle \backslash \triangle_{\frac{1}{2}}} \mu(z) d x d y+\iint_{\triangle_{\frac{1}{2}}} \frac{\rho z}{2|z|+\rho \bar{z}} d x d y\right| \\
& \geqslant \frac{1}{\pi}\left\{\left|\iint_{\triangle_{\frac{1}{2}}} \frac{\rho z}{2|z|+\rho \bar{z}} d x d y\right|-\frac{3}{4} \pi k\right\} .
\end{aligned}
$$


由于

$$
\begin{aligned}
\left|\iint_{\triangle_{\frac{1}{2}}} \frac{\rho z}{2|z|+\rho \bar{z}} d x d y\right| & =\left|\int_{0}^{\frac{1}{2}} r d r \int_{|z|=r} \frac{\rho z}{2|z|+\rho \bar{z}} \frac{d z}{\mathrm{i} z}\right| \\
& =\left|\int_{0}^{\frac{1}{2}} r d r \int_{|z|=r} \frac{\rho z}{2 r z+\rho r^{2}} d z\right| \\
& =\left|\int_{0}^{\frac{1}{2}} \frac{1}{2} \pi \rho^{2} r d r\right|=\frac{1}{16} \pi \rho^{2},
\end{aligned}
$$

根据 (3.3) 和 (3.4) 式以及假定 $\|\mu\|_{\infty}=k<\frac{1}{28} \rho^{2}$, 得到

$$
\|\nu\| \geqslant \frac{1}{\pi}\left\{\frac{1}{16} \pi \rho^{2}-\frac{3}{4} \pi k\right\}>\frac{28}{16} k-\frac{3}{4} k=k \geqslant b([\nu]),
$$

因此 $[\nu]_{B(\triangle)}$ 是一个无限小 Strebel 点, 定理证明完毕.

\section{4 定理 2 的证明}

为证明定理 2 , 需要介绍 Reich 构造定理以及主要不等式.

Reich 构造定理 ${ }^{[13]}$ 令 $A$ 是 $\triangle$ 的紧子集, $A$ 中至少包含两个点并且 $\triangle \backslash A$ 是双连通 的, 则存在一个函数 $\alpha(z) \in L^{\infty}(\triangle)$ 和序列 $\varphi_{n}(z) \in A(\triangle)(n \in \mathbb{N})$, 使得它们满足条件

$$
\begin{aligned}
& |\alpha(z)|=\left\{\begin{array}{l}
0, z \in A, \\
1, \text { a.e. } z \in \Delta \backslash A,
\end{array}\right. \\
& \lim _{n \rightarrow \infty}\left\{\left\|\varphi_{n}\right\|-\lambda_{\alpha}\left[\varphi_{n}\right]\right\}=0, \\
& \lim _{n \rightarrow \infty}\left|\varphi_{n}(z)\right|=\infty, \text { a.e. } z \in \triangle \backslash A,
\end{aligned}
$$

以及在集合 $A$ 上成立

$$
\varphi_{n}(z) \rightrightarrows 0,
$$

其中 $\lambda_{\alpha}[\varphi]=\operatorname{Re} \iint_{\triangle} \alpha(z) \varphi(z)$. 需要指出条件 (4.4) 是蕴涵在 Reich 构造定理的证明过程中 的.

主要不等式 ${ }^{[3]}$ 令 $f$ 和 $g$ 是 $\triangle$ 到自身的拟共形映射且满足 $\left.f\right|_{\partial \triangle}=\left.g\right|_{\partial \triangle}$, 则对任意 $\varphi \in A(\triangle)$, 成立

$$
\|\varphi\| \leqslant \iint_{\triangle}|\varphi(z)| \frac{\left|1-\mu \frac{\varphi}{|\varphi|}\right|^{2}}{1-|\mu|^{2}} \frac{\left|1+\mu \frac{\widetilde{\nu}(f)}{\widetilde{\mu}(f)} \frac{\varphi}{|\varphi|} \frac{1-\bar{\mu} \frac{\varphi}{\varphi \varphi} \mid}{1-\mu \frac{\varphi}{\mid \varphi}}\right|^{2}}{1-|\widetilde{\nu}(f)|^{2}} d x d y,
$$

其中 $\mu, \widetilde{\mu}$ 和 $\widetilde{\nu}$ 分别是 $f, f^{-1}$ 和 $g^{-1}$ 的 Beltrami 系数.

在文献 [15] 中 Reich 证明了 (4.5) 式具有下面的等价形式:

$$
\iint_{\triangle} L(z)|\varphi(z)| d x d y \leqslant \operatorname{Re} \iint_{\triangle} R(z)\left(|\varphi(z)|-\frac{\mu(z)}{|\mu(z)|} \varphi(z)\right) d x d y,
$$

其中

以及

$$
L(z)=\frac{|\widetilde{\mu}|^{2}-|\widetilde{\nu}|^{2}+(1-|\widetilde{\mu}|)\left(|\widetilde{\mu}|-\operatorname{Re} \frac{\overline{\widetilde{\nu}} \widetilde{\widetilde{\mu}}}{\widetilde{\mu} \mid}\right)}{(1+|\widetilde{\mu}|)\left(1-|\widetilde{\nu}|^{2}\right)}, \quad R(z)=\frac{\overline{\widetilde{\mu}}}{|\widetilde{\mu}|} \frac{(1-\overline{\widetilde{\nu}} \widetilde{\mu})(\widetilde{\mu}-\widetilde{\nu})}{\left(1-|\widetilde{\mu}|^{2}\right)\left(1-|\widetilde{\nu}|^{2}\right)}
$$

$$
\widetilde{\mu}(z)=\mu_{f^{-1}}(f(z)), \quad \widetilde{\nu}(z)=\mu_{g^{-1}}(f(z)) .
$$


事实上, 我们的证明将用到的是 (4.6) 式.

引理 2 假设 $J \subset \triangle$ 是一个 Jordan 区域, 记 $A=\bar{J} \subset \triangle$. 令 $\alpha(z)$ 和 $\varphi_{n}(z) \in A(\triangle)(n \in$ $\mathbb{N})$ 为 Reich 构造定理中的函数和序列, 并记 $\mu(z)=k \alpha(z)$, 其中 $k<1$ 是正常数. 令

$$
\nu(z)= \begin{cases}\mu(z), & z \in \Delta \backslash A, \\ \eta(z), & z \in A,\end{cases}
$$

其中 $\eta(z) \in M(J)$. 如果 $k_{0}\left([\nu]_{T(\triangle)}\right) \leqslant k$, 则对 $[\nu]_{T(\triangle)}$ 中的任意极值 Beltrami 系数 $\chi(z)$, 在 $\triangle \backslash A$ 都几乎处处成立 $\chi(z)=\nu(z)$.

证明从 $\nu$ 的定义以及式子 (4.2) 和 (4.4), 我们得到

$$
\delta_{n}=\iint_{\triangle \backslash A}\left\{k\left|\varphi_{n}\right|-\operatorname{Re}\left[\mu \varphi_{n}\right]\right\} d x d y \rightarrow 0, \quad n \rightarrow \infty .
$$

假设 $\chi(z)$ 为 $[\nu]_{T(\Delta)}$ 中的极值 Beltrami 系数并且 $\|\chi\|_{\infty}=k_{0}\left([\nu]_{T(\Delta)}\right) \leqslant k$, 令 $f(z)$ 和 $g(z)$ 是规范化的 $\triangle$ 到自身的拟共形映射且分别以 $\nu(z)$ 和 $\chi(z)$ 为复特征. 记 $\widetilde{\mu}(z)=\mu_{f^{-1}}(f(z))$, $\widetilde{\nu}(z)=\mu_{g^{-1}}(f(z))$, 则

$$
|\widetilde{\mu}(z)|=|\nu(z)|=|\mu(z)|=k, \quad|\widetilde{\nu}(z)|=|\chi(z)| \leqslant k \quad(z \in \triangle \backslash A) .
$$

应用 (4.6) 式以及 $(4.4)$ 式, 我们得到

$$
\iint_{\triangle \backslash A} L(z)\left|\varphi_{n}(z)\right| d x d y \leqslant \operatorname{Re} \iint_{\triangle \backslash A} R(z)\left(\left|\varphi_{n}(z)\right|-\frac{\mu(z)}{|\mu(z)|} \varphi_{n}(z)\right) d x d y+\tau_{n},
$$

且 $\lim _{n \rightarrow \infty} \tau_{n}=0$. 根据 (4.8) 式, 当 $z \in \triangle \backslash A$ 时, 可以得到下面的估计:

以及

$$
\begin{aligned}
L(z) & =\frac{\left.|\widetilde{\mu}|^{2}-|\widetilde{\nu}|^{2}+(1-|\widetilde{\mu}|)\left(|\widetilde{\mu}|-\operatorname{Re} \frac{\overline{\bar{\nu}} \widetilde{\mu}}{|\widetilde{\mu}|}\right)\right)}{(1+|\widetilde{\mu}|)\left(1-|\widetilde{\nu}|^{2}\right)} \\
& \geqslant \frac{(1-k)\left(k-k \operatorname{Re} \frac{\overline{\bar{\nu}}}{\overline{\widetilde{\mu}}}\right)}{1+k} \\
& =\frac{k(1-k)}{1+k}\left(1-\operatorname{Re} \frac{\overline{\widetilde{\nu}}}{\overline{\widetilde{\mu}}}\right)
\end{aligned}
$$

$$
|R(z)|=\left|\frac{\overline{\widetilde{\mu}}}{|\widetilde{\mu}|} \frac{(1-\overline{\widetilde{\nu}} \widetilde{\mu})(\widetilde{\mu}-\widetilde{\nu})}{\left(1-|\widetilde{\mu}|^{2}\right)\left(1-|\widetilde{\nu}|^{2}\right)}\right| \leqslant \frac{1+k^{2}}{\left(1-k^{2}\right)^{2}}|\widetilde{\mu}-\widetilde{\nu}| .
$$

由于

$$
\begin{aligned}
|\widetilde{\mu}-\widetilde{\nu}|^{2} & =|\widetilde{\mu}|^{2}+|\widetilde{\nu}|^{2}-2 \operatorname{Re} \widetilde{\mu} \bar{\nu}=k^{2}+|\widetilde{\nu}|^{2}-2 k^{2} \operatorname{Re} \frac{\overline{\widetilde{\nu}}}{\overline{\widetilde{\mu}}} \\
& \leqslant 2 k^{2}-2 k^{2} \operatorname{Re} \frac{\overline{\widetilde{\nu}}}{\overline{\widetilde{\mu}}}=2 k^{2}\left(1-\operatorname{Re} \frac{\overline{\widetilde{\nu}}}{\overline{\widetilde{\mu}}}\right),
\end{aligned}
$$

由 (4.10) 和 (4.12) 式, 得到

$$
L(z) \geqslant \frac{1-k}{2 k(1+k)}|\widetilde{\mu}-\widetilde{\nu}|^{2} .
$$

由 (4.13) 式, 得到 (4.9) 式左边的一个下界为

$$
\frac{1-k}{2 k(1+k)} \iint_{\triangle \backslash A}|\widetilde{\mu}-\widetilde{\nu}|^{2}\left|\varphi_{n}\right| d x d y,
$$

根据 (4.11) 式就得到了 (4.9) 式右边的一个上界为

$$
\frac{1+k^{2}}{k\left(1-k^{2}\right)^{2}} \iint_{\triangle \backslash A}|\widetilde{\mu}-\widetilde{\nu}||k| \varphi_{n}\left|-\mu \varphi_{n}\right| d x d y .
$$


令

$$
I_{n}=\iint_{\triangle \backslash A}|\widetilde{\mu}-\widetilde{\nu}|^{2}\left|\varphi_{n}\right| d x d y
$$

因为对任意复数 $w$ 都成立 ||$w|-w|^{2}=2|w|(|w|-\operatorname{Re} w)$, 利用 Schwarz 不等式, 我们得到

$$
\begin{aligned}
\iint_{\triangle \backslash A}|\widetilde{\mu}-\widetilde{\nu}||k| \varphi_{n}\left|-\mu \varphi_{n}\right| d x d y & =\left.\iint_{\triangle \backslash A}|\widetilde{\mu}-\widetilde{\nu}|\left|\varphi_{n}\right|^{\frac{1}{2}}|k| \varphi_{n}\right|^{\frac{1}{2}}-\mu \frac{\varphi_{n}}{\left|\varphi_{n}\right|^{\frac{1}{2}}} \mid d x d y \\
& \leqslant \sqrt{\iint_{\triangle \backslash A}|\widetilde{\mu}-\widetilde{\nu}|^{2}\left|\varphi_{n}\right| d x d y} \sqrt{\left.|k| \varphi_{n}\right|^{\frac{1}{2}}-\left.\mu \frac{\varphi_{n}}{\left|\varphi_{n}\right|^{\frac{1}{2}}}\right|^{2} d x d y} \\
& =\sqrt{2 k I_{n} \delta_{n}} .
\end{aligned}
$$

所以, 由 (4.9) 和 (4.14)-(4.16) 式得

$$
\frac{1-k}{2 k(1+k)} I_{n} \leqslant \frac{1+k^{2}}{k\left(1-k^{2}\right)^{2}} \sqrt{2 k I_{n} \delta_{n}}+\tau_{n} .
$$

由于 $\lim _{n \rightarrow \infty} \tau_{n}=0$ 和 (4.7) 式, 则 $\lim _{n \rightarrow \infty} I_{n}=0$. 根据 (4.3) 式和 Fatou 引理, 得到

$$
\widetilde{\mu}(z)=\widetilde{\nu}(z) \quad \text { a.e. } z \in \triangle \backslash A \text {. }
$$

所以 $g^{-1} \circ f(z)$ 在 $\triangle \backslash A$ 内是共形映射, 又由于 $g^{-1} \circ f(z)$ 在 $\partial \triangle$ 上是恒等映射, 所以当 $z \in \triangle \backslash A$ 时 $g^{-1} \circ f(z)=\mathrm{id}$, 即

$$
f(z)=g(z), \quad z \in \triangle \backslash A,
$$

所以在 $\triangle \backslash A$ 上几乎处处成立 $\chi(z)=\nu(z)$, 证明完毕.

姚国武在文献 [11] 中证明了以下引理, 该引理的证明也可以参考文献 $[12,16]$.

引理 3 令 $A \subset \triangle$ 是 $\triangle$ 内的一个 Jordan 区域且 $\triangle \backslash \bar{A}$ 连通, $[\mu]_{T(\triangle)}=[\nu]_{T(\triangle)}$. 如果 在 $\triangle \backslash \bar{A}$ 中几乎处处成立 $\mu(z)=\nu(z)$, 则 $\left[\left.\mu\right|_{A}\right]_{T(A)}=\left[\left.\nu\right|_{A}\right]_{T(A)}$.

引理 $4 \quad[t z]_{B\left(\triangle_{\frac{1}{2}}\right)}=[0]_{B\left(\triangle_{\frac{1}{2}}\right)}$, 其中 $t$ 是一个常数.

证明 对任意 $\varphi(z) \in A\left(\triangle_{\frac{1}{2}}\right)$, 有

$$
I=\iint_{\triangle_{\frac{1}{2}}} t z \varphi(z) d x d y=\int_{0}^{\frac{1}{2}} r d r \int_{|z|=r} \varphi(z) t z \frac{d z}{i z}=\int_{0}^{\frac{1}{2}} r d r \int_{|z|=r} \frac{t}{i} \varphi(z) d z .
$$

由 Cauchy 积分定理可以得到对任意 $\varphi(z) \in A\left(\triangle_{\frac{1}{2}}\right)$ 都成立 $I=0$.

引理 $5 \quad k_{0}\left([t z]_{T\left(\triangle_{\frac{1}{2}}\right)}\right)>0$, 其中 $0<t<2$.

证明 令 $g(z)=z e^{t \bar{z}}, z \in \triangle_{\frac{1}{2}}$, 则 $\mu_{g}(z)=t z$. 因为 $\left\|\mu_{g}(z)\right\|_{\infty}<1$, 所以 $g(z)$ 是 $\triangle_{\frac{1}{2}}$ 到 $g\left(\triangle_{\frac{1}{2}}\right)$ 的拟共形映射且以 $t z$ 为复特征. 如果 $k_{0}\left([t z]_{T\left(\triangle_{\frac{1}{2}}\right)}\right)=0$, 则存在一个 $g\left(\triangle_{\frac{1}{2}}\right)$ 到 $\triangle_{\frac{1}{2}}$ 的共形映射, 使得当 $|z|=\frac{1}{2}$ 时 $f \circ g(z) \equiv \mathrm{id}$, 即当 $|z|=\frac{1}{2}$ 时 $f^{-1}(z)=z e^{\frac{t}{4 z}}$. 因为 $f^{-1}(z)$ 是 $\triangle_{\frac{1}{2}}$ 到 $g\left(\triangle_{\frac{1}{2}}\right)$ 的共形映射, 根据解析函数延拓的唯一性, 我们得到对任意 $z \in \triangle_{\frac{1}{2}}$ 成立 $f^{-1}(z)=z e^{\frac{t}{4 z}}$. 但是 $f^{-1}(z)$ 在 $z=0$ 有一个奇点, 这就产生了矛盾. 所以 $k_{0}\left([t z]_{T\left(\triangle_{\frac{1}{2}}\right)}\right)>0$, 证明完毕.

根据上述一些引理, 我们现在来证明定理 2 .

定理 2 的证明 记 $A=\bar{\triangle}_{\frac{1}{2}}$. 取 $\alpha(z)$ 为 Reich 构造定理中的函数, $t$ 为区间 $(0,2)$ 之间 的一个常数, $\mu(z)=k \alpha(z)$, 其中 $0<k<k_{0}\left([t z]_{T\left(\triangle_{\frac{1}{2}}\right)}\right), k$ 的存在性可以由引理 5 保证. 根据 Reich 构造定理, 知 $[\mu]_{B(\triangle)}$ 是一个无限小非 Strebel 点. 
作

$$
\nu(z)= \begin{cases}\mu(z), & z \in \triangle \backslash \bar{\triangle}_{\frac{1}{2}}, \\ t z, & z \in \bar{\triangle}_{\frac{1}{2}} .\end{cases}
$$

则对任意 $\varphi \in A(\triangle)$, 由引理 4 得到 $\iint_{\triangle}(\nu(z)-\mu(z)) \varphi(z) d x d y=\iint_{\triangle_{\frac{1}{2}}} t z \varphi(z) d x d y=0$, 所 以 $[\mu]_{B(\triangle)}=[\nu]_{B(\triangle)}$. 下面证明 $[\nu]_{T(\triangle)}$ 是一个 Strebel 点.

假设 $\chi(z)$ 为 $[\nu]_{T(\Delta)}$ 中的一个极值 Beltrami 系数. 如果 $k_{0}\left([\nu]_{T(\Delta)}\right) \leqslant k$, 那么根据引理 2 和 3 , 得到

$$
\left[\left.\chi\right|_{\triangle_{\frac{1}{2}}}\right]_{T\left(\triangle_{\frac{1}{2}}\right)}=\left[\left.\nu\right|_{\triangle_{\frac{1}{2}}}\right]_{T\left(\triangle_{\frac{1}{2}}\right)}=[t z]_{T\left(\triangle_{\frac{1}{2}}\right)} .
$$

则 $k \geqslant k_{0}\left([\nu]_{T(\triangle)}\right)=\|\chi\|_{\infty} \geqslant\left\|\left.\chi\right|_{\triangle_{\frac{1}{2}}}\right\|_{\infty} \geqslant k_{0}\left([t z]_{T\left(\triangle_{\frac{1}{2}}\right)}\right)$, 这与假设 $0<k<k_{0}\left([t z]_{T\left(\triangle_{\frac{1}{2}}\right)}\right)$ 矛盾, 因此 $k_{0}\left([\nu]_{T(\Delta)}\right)>k$. 此外, 根据 $h\left([\nu]_{T(\Delta)}\right)$ 的定义很容易知道 $h\left([\nu]_{T(\Delta)}\right) \leqslant k$, 所以 $h\left([\nu]_{T(\triangle)}\right)<k_{0}\left([\nu]_{T(\triangle)}\right)$, 这就证明了 $[\nu]_{T(\triangle)}$ 是一个 Strebel 点. 定理证明完毕.

\section{5 定理 3 和 4 的证明}

在 Reich 构造定理中, $\triangle \backslash A$ 需要是双连通的. 从该定理的证明中, 我们发现 $\triangle \backslash A$ 为双 连通这个条件不是必要的. 鉴于发现这点, 姚国武将 Reich 构造定理推广到一般多连通的情 形.

构造定理 ${ }^{[12]}$ 令 $A=\bigcup_{i=1}^{m} A_{i}$, 每个 $A_{i}$ 都是 $\triangle$ 的一个紧子集且至少包含两点, 此外 $A_{i}$ 还满足 $\triangle \backslash A_{i}$ 是双连通的并且 $A_{i} \cap A_{j}=\emptyset(i \neq j)$, 则存在一个函数 $\alpha(z) \in L^{\infty}(\triangle)$ 和序 列 $\varphi_{n} \in A(\triangle)(n \in \mathbb{N})$ 也满足 (4.1)-(4.4) 式.

取 $\mu(z)=k \alpha(z), A_{i} \subset \triangle(i=1,2, \ldots, m)$ 是 $m$ 个连通 Jordan 区域, 使得 $\overline{A_{i}} \cap \overline{A_{j}}=\emptyset(i \neq j)$. 根据上述构造定理, 姚国武构造了下列的 Beltrami 系数:

$$
\eta(z)= \begin{cases}\mu(z), & z \in \Delta \backslash \bigcup_{1}^{m} \overline{A_{i}}, \\ \beta_{1}(z), & z \in \overline{A_{1}}, \\ \beta_{2}(z), & z \in \overline{A_{2}}, \\ \beta_{i}(z), & z \in \overline{A_{i}}, 3 \leqslant i \leqslant m,\end{cases}
$$

其中 $\beta_{1}(z)$ 在 $T\left(A_{1}\right)$ 中唯一极值且 $\left\|\beta_{1}(z)\right\|_{\infty}=k$ 以及 $\left|\beta_{1}(z)\right|$ 在 $A_{1}$ 上不是常数, 此外 $\beta_{2}(z) \in M\left(A_{2}\right)$ 且 $\left\|\beta_{2}\right\|_{\infty}<k, \beta_{i}(z) \in M\left(A_{i}\right)$ 且 $\left\|\beta_{i}(z)\right\|_{\infty} \leqslant k$. 姚国武 ${ }^{[12]}$ 证明了 $[\eta]_{T(\triangle)}$ 包 含无穷多个极值 Beltrami 系数, 但是 $[\eta]_{T(\triangle)}$ 中不包含常数模极值 Beltrami 系数. 对同样的 $\eta(z)$, 姚国武 ${ }^{[12]}$ 也证明了 $[\eta]_{B(\triangle)}$ 包含无穷多个无限小极值 Beltrami 系数, 但是 $[\eta]_{B(\triangle)}$ 中 不包含常数模无限小极值 Beltrami 系数.

定理 3 的证明 取 $E=\left\{z:\left|z-\frac{3}{4}\right|<\frac{1}{8}\right\}, \mu(z)=k \alpha(z)$, 令

$$
\eta(z)= \begin{cases}\beta(z), & z \in \bar{E}, \\ 0, & z \in \bar{\triangle}_{\frac{1}{2}}, \\ \mu(z), & z \in \triangle \backslash\left(\bar{E} \cup \bar{\triangle}_{\frac{1}{2}}\right),\end{cases}
$$

其中 $\beta(z)$ 在 $T(E)$ 上唯一极值, $\|\beta(z)\|_{\infty}=k$ 且 $|\beta(z)|$ 在 $E$ 上不是常数. 由姚国武 ${ }^{[12]}$ 的结 论, 我们知道 $[\eta]_{T(\triangle)}$ 中包含无穷多个极值 Beltrami 系数, 但是 $[\eta]_{T(\triangle)}$ 中不包含常数模极值 
Beltrami 系数. 类似于定理 1 的证明, 取

$$
\nu(z)=\left\{\begin{array}{l}
\eta(z), z \in \Delta \backslash \bar{\triangle}_{\frac{1}{2}}, \\
\frac{\rho z}{2|z|+\rho \bar{z}}, z \in \bar{\triangle}_{\frac{1}{2}},
\end{array}\right.
$$

则 $[\nu]_{T(\triangle)}=[\eta]_{T(\triangle)}$. 当 $\rho \neq 0$ 以及 $k$ 充分小 $\left(0<k<\frac{1}{28} \rho^{2}\right)$ 的时候, 类似于定理 1 的证明, 知 $[\nu]_{B(\triangle)}$ 是一个无限小 Strebel 点, 因此 $[\nu]_{B(\triangle)}$ 包含唯一极值 Teichmüller Beltrami 系数. 所以我们构造了一个 Beltrami 系数 $\nu$, 使得 $[\nu]_{T(\Delta)}$ 中不包含常数模极值 Beltrami 系数, 但 是 $[\nu]_{B(\triangle)}$ 中包含一个唯一极值 Teichmüller Beltrami 系数, 证明完毕.

定理 4 的证明 取 $\eta(z)$ 为 (5.2) 式中所定义的, 由姚国武 ${ }^{[12]}$ 的结论, 知 $[\eta]_{B(\triangle)}$ 中不 包含常数模极值 Beltrami 系数. 类似于定理 2 的证明, 令

$$
\nu(z)=\left\{\begin{array}{l}
\eta(z), z \in \triangle \backslash \bar{\triangle}_{\frac{1}{2}}, \\
t_{0} z, z \in \bar{\triangle}_{\frac{1}{2}},
\end{array}\right.
$$

则 $[\nu]_{T(\triangle)}=[\eta]_{T(\triangle)}$. 当 $0<t_{0}<2, k$ 充分小 $\left(0<k<k_{0}\left[t_{0} z\right]_{T\left(\triangle_{\frac{1}{2}}\right)}\right)$ 的时候, 类似于定理 2 的证明, 我们可以得到 $[\nu]_{T(\Delta)}$ 是一个 Strebel 点, 因此 $[\nu]_{T(\triangle)}$ 包含唯一极值 Teichmüller Beltrami 系数. 因此我们构造了一个 Beltrami 系数 $\nu$, 使得 $[\nu]_{B(\triangle)}$ 中不包含常数模极值 Beltrami 系数, 但是 $[\nu]_{T(\triangle)}$ 中包含唯一极值 Teichmüller Beltrami 系数, 证明完毕.

在定理 1 和 2 中, 对一些特殊的非 Strebel 点 $[\mu]_{T(\Delta)}$, 我们构造了 $\nu \in[\mu]_{T(\Delta)}$, 使得 $[\nu]_{B(\triangle)}$ 为无限小 Strebel 点. 同样对一些特殊的无限小非 Strebel 点 $[\mu]_{B(\triangle)}$, 我们构造了 $\nu \in[\mu]_{B(\triangle)}$, 使得 $[\nu]_{T(\triangle)}$ 为 Strebel 点. 但是还不知道上述结论对一般的非 Strebel 点或者 无限小非 Strebel 点是否成立. 作为本文的结束, 我们提出以下问题:

问题 令 $[\mu]_{T(\triangle)}$ 为一个非 Strebel 点, 是否一定存在 $\nu \in[\mu]_{T(\Delta)}$, 使得 $[\nu]_{B(\triangle)}$ 为一个 无限小 Strebel 点? 令 $[\mu]_{B(\triangle)}$ 为一个无限小非 Strebel 点, 是否一定存在 $\nu \in[\mu]_{B(\triangle)}$, 使得 $[\nu]_{T(\triangle)}$ 为一个 Strebel 点?

致谢作者衰心感谢姚国武寄来文章 $[11,12]$, 也同样感谢审稿人提出的宝贵意见.

\section{参考文献}

1 Hamilton R S. Extremal quasiconformal mappings with prescribed boundary values. Trans Amer Math Soc, 138: 399-406 (1969)

2 Krushkal S. Extremal quasiconformal mappings. Sib Math J, 10: 411-418 (1969)

3 Reich E, Strebel K. Extremal quasiconformal mappings with given boundary values. In: Contribution to Analysis (A Collections of Papers Dedicated to Lipman Bers), New York: Academic Press, 1974, 375-392

4 Božin V, Lakic N, Marković V, et al. Unique extremality. J Anal Math, 75: 299-338 (1998)

5 Lakic N. Strebel points. Contemp Math, 211: 417-431 (1997)

6 Strebel K. On the existence of extremal Teichmüller mappings. J Anal Math, 30: 464-480 (1976)

7 Strebel K. Point shift differentials and extremal quasiconformal mappings. Ann Acad Sci Fenn Math, 23: 475-494 (1998)

8 Earle C J, Kra I, Krushkal S. Holomorphic motions and Teichmüller spaces. Trans Amer Math Soc, 343: 927-948 (1994) 
9 Earle C J, Li Z. Isometrically embedded polydisks in infinite dimensional Teichmüller spaces. J Geom Anal, 9: $51-71$ (1999)

10 Li Z. Strebel differentials and Hamilton sequences. Sci China Ser A-Math, 44: 969-979 (2001)

11 Yao G. Is there always an extremal Teichmüller mapping. J Anal Math, 94: 363-375 (2004)

12 Yao G. Existence of extremal Beltrami coefficients with non-constant modulus. preprint

13 Reich E. The unique extremality counterexample. J Anal Math, 75: 339-347 (1998)

14 Shen Y. On Teichmüller geometry. Complex Variables Theory Appl, 44: 73-83 (2001)

15 Reich E. On criteria for unique extremality of Teichmüller mappings. Ann Acad Sci Fenn Ser A I Math, 6: 289-301 (1981)

16 Yao G. Unique extremality, local extremality and extremal non-decreasable dilatations. Bull Aust Math Soc, 75: 321-329 (2007) 